\title{
A multiscale numerical model for structures with internal frictional contacts
}

\author{
K. Truyaert ${ }^{1[0000-0001-5885-308 X]}$, V. Aleshin ${ }^{2[0000-0002-8933-3367]}$, \\ $\underline{\text { S. Delrue }}^{1[0000-0001-7439-1604]}$, and K. Van Den Abeele ${ }^{1[0000-0003-0327-6478]}$ \\ 1 Wave Propagation and Signal Processing Research Group, \\ KU Leuven Kulak, Kortrijk, Belgium, \\ steven.delrue@kuleuven.be \\ 2 Joint International Laboratory LICS/LEMAC, IEMN, \\ Villeneuve d'Ascq CEDEX, France
}

\begin{abstract}
Many engineering applications are related to or deal with materials and systems having internal frictional contacts in their structure. The effects induced by these contacts, such as friction-induced heat generation, wear, nonlinear vibrations, etc., can be significant and cannot be ignored in numerical models. However, friction models are computationally cumbersome because they often require implicit data exchange procedures to describe the contact evolution. Moreover, detailed meshing of the contact zone is needed to cover the microgeometry (roughness). Here, an alternative model is proposed, based on a semi-analytical method of contact mechanics, called the Method of Memory Diagrams (MMD), that allows for an automated explicit calculation of the hysteretic frictional contact response. The key strength of the method is that it uses a multiscale approach in which mesoscopic cells, containing a section of the frictional contact, are introduced to resolve the stress and displacement fields at the contact interface into a single loaddisplacement relationship. Hence, the essential constitutive information of the contact is supplied to the macroscale model by the mesoscopic cells, drastically simplifying the account for rough contacts and avoiding microscopic meshing of the contact geometry. The MMD contact model is directly integrated into a Finite Element Modeling (FEM) environment enabling the study of the dynamic behavior of structures with frictional interfaces. The potential of the proposed model for engineering applications will be demonstrated by simulating the contact behavior of a dynamically excited frictional contact and by linking this behavior to friction-induced effects such as nonlinear vibrations and heat production.
\end{abstract}

Keywords: Computational modeling, Contact mechanics, Method of Memory Diagrams, Acoustic wave propagation, Thermosonics

\section{Introduction}

Internal frictional contacts occur in many engineering applications. Hence, realistic modeling of these contacts is of crucial importance. In the present study 
we propose a contact mechanical model based on certain compromises related to the level of complexity and realism.

One of the most simple models for internal contacts are based on stiffness asymmetry by using different elastic moduli for normal compression and tension. However, such elementary models are unable to represent one of the major mechanisms in mechanical contacts: friction. Introducing friction into the models drastically increases the complexity level and makes the system hysteretic and memory-dependent.

Frictional contact models often rely on purely numerical approaches of contact mechanics [1]. This allows to take all particular interaction laws and movement types into account. However, the difficulty related to this approach is in the implicit character of the procedure. Accepting the Coulomb friction law for representing interactions of flat contact faces [2] does not provide an explicit link between contact loads and displacements. Instead, an iterative procedure is necessary in which some trial contact face displacements are assumed and iteratively adjusted to satisfy the Coulomb conditions. Such methods are intensively developed despite the high computational expenses arising due to the implicit calculations of complex hysteretic dependencies.

The compromise we propose is based on a semi-analytical model in contact mechanics that can also be referred to as a generalized Hertz-Mindlin approach. The classical Hertz-Mindlin solution [3] is the load-displacement relationship for a tangential shift of two spheres with friction, excited by a number of specific loading protocols, such as constant oblique loading, oblique loading-unloadingreloading series, oscillatory oblique loading, etc. Here the solution is extended to the case of non-spherical geometries, including rough surfaces. The contact surfaces will be represented as a collection of fragments (called mesoscopic cells) and the influence of microgeometry will be integrated in the response of the mesoscopic cells using a simple memory function that uses mean values for loads and displacements instead of the complex stress and displacement fields in the contact zone. Moreover, the instantaneous friction-induced loss of mechanical energy is also easily calculated. Note also that the account for surface roughness in the proposed model increases the efficiency of the code as no implicit data exchange procedures are required to describe the contact evolution.

The full approach is discussed in more detail in the next sections and the potential of the method is illustrated by means of an example of ultrasonic wave-crack interaction.

\section{Frictional contact model formulation}

In this section, the model used to describe the particular behavior of a dynamically excited contact is formulated. First, the mechanical contact problem is discussed. Then, the model is extended to also consider friction-induced heat generation. 


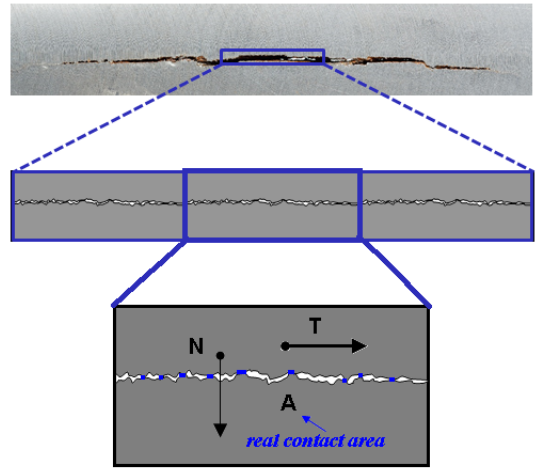

Fig. 1. Division of a crack into mesoscopic cells.

\subsection{Mechanical contact problem}

The proposed model of frictional contacts takes asperities into account, making the consideration more complicated. However, this difficulty is easily dealt with when using the property that every complex contact profile with asperities can be replaced by an equivalent axisymmetric, convex contact profile [4]. It allows to separate a crack into a number of mesoscopic cells each hosting a large number of microscopic features, as illustrated in Figure 1. The cell size is chosen smaller than the characteristic scale of the macroscopic elastic field, such that the mescoscopic level description includes average stresses or loads per unit area, $N$ and $T$, and displacements $a$ and $b$, that are linked to each other by way of a particular relationship that can be theoretically derived for axisymmetric, convex contact profiles. The full contact mechanics problem is solved by describing the contact in each cell separately. Since roughness is only considered at the microscopic level, crack faces at the mesoscopic level can be modeled as flat in the final numerical model.

Mechanical contact between two non-flat surfaces can evolve in one of the following three states: contact loss, total sliding and partial slip. The latter only occurs due to the presence of surface relief, since surface points having different heights are compressed differently under a normal loading scenario. In combination with the applied shear load, these points will have a different tangential behavior (either stick or slip). The particular case of partial slip will be discussed first, before introducing the (more trivial) cases of contact loss and full sliding into the model.

The Method of Memory Diagrams (MMD) links the normal and tangential loads, $N$ and $T$, to the normal and tangential displacements, $a$ and $b$, through an internal memory function that is responsible for all hysteretic effects. The theory behind MMD is based on the Reduced Elastic Friction Principle (REFP, $[5,6])$ that allows to express the tangential variables of the axisymmetric contact 
as function of the normal ones in the following way:

$$
\left\{\begin{array}{l}
b=\theta \mu(a-q) \\
T=\mu(N(a)-N(q))
\end{array},\right.
$$

where $\mu$ is the friction coefficient, $\theta$ is a material parameter based on Poisson's ratio $\nu$ :

$$
\theta=\frac{2-\nu}{2(1-\nu)},
$$

$N(a)$ is the relation between the normal force and normal displacement which is considered to be known either by experimental observations or from theoretical calculations using contact mechanics formulations, and $q$ is a normal indentation parameter that can be used to separate the stick from the slip region. Equation (1) can be rewritten following the description of MMD:

$$
\left\{\begin{array}{l}
b=\theta \mu \int_{0}^{a} D(\alpha) \mathrm{d} \alpha \\
T=\left.\mu \int_{0}^{a} D(\alpha) \frac{\mathrm{d} N}{\mathrm{~d} a}\right|_{a=\alpha} \mathrm{d} \alpha
\end{array} .\right.
$$

Here, the memory diagram $D(\alpha)$, which is a function that contains all memory information present in the contact system, is introduced. In previous papers about MMD, rules are defined to update the memory function following the history of drive parameters (here displacement $b$ ) and to calculate the output (here tangential load $T$ ) that is needed to construct the required hysteretic loaddisplacement relationship for the partial slip phase $[7,8]$.

The partial slip state remains as long as Coulomb's frictional description holds (i.e. $|T|<\mu N$, or in displacement terms $|b|<\theta \mu a$ ). When $|T|$ reaches $\mu N$, or similarly when $|b|$ reaches $\theta \mu a$, partial slip becomes total sliding. To account for total sliding, the tangential displacement parameter $b$ is split into two components [8]:

$$
b=b_{0}+\tilde{b} .
$$

Here, $b_{0}$ is the total sliding distance, which is the current tangential distance between two points belonging to opposite faces and having the same tangential coordinate in the unperturbed geometry, while $\tilde{b}$ contains information about the current partial slip state as it takes into account the ability of asperities to recede under tangential load. Hence, the latter is the parameter that is needed to update the memory function. The solutions for the three different contact states (contact loss, partial slip and total sliding) can now be described as follows:

- In case of contact loss (i.e. $a<0)$, no contact interaction is present $(N=$ $T=0)$ and asperities remain unstrained $(\tilde{b}=0)$. Hence, $b_{0}=b$ in this case. The memory function equals zero for all $\alpha$.

- During partial slip, $a \geq 0$ and $|\tilde{b}|<\theta \mu a$. The tangential load is in this case calculated according to Equation (3), using $b=\tilde{b}$. The shape of the memory function will depend on the loading history. 
Table 1. The three possible contact states in the model of contact defects with rough surfaces, with for each state the conditions under which the contact state occurs, the solutions for the tangential displacement components $b_{0}$ and $\tilde{b}$, the solutions for the normal and tangential forces $N$ and $T$, and the memory diagram $D(\alpha)$ (Taken from $[9])$.

\begin{tabular}{|c|c|c|c|}
\hline Crack state & If & Then & Memory diagram \\
\hline (i) Contact loss & $a(t)<0$ & $\begin{array}{c}\tilde{b}(t)=0 \\
b_{0}(t)=b(t) \\
T(t)=N(t)=0\end{array}$ & $\uparrow_{-1}^{1} \uparrow^{D(\alpha)}$ \\
\hline (ii) Total sliding & $\begin{array}{c}a(t) \geq 0 \\
|\tilde{b}(t-\Delta t)| \geq \theta \mu a(t)\end{array}$ & $\begin{array}{c}\tilde{b}(t)= \pm \theta \mu a(t) \\
b_{0}(t)=b(t)-\tilde{b}(t) \\
T(t)= \pm \mu N(t)\end{array}$ & $11 \mid$ \\
\hline (iii) Partial slip & $\begin{aligned} a(t) & \geq 0 \\
|\tilde{b}(t-\Delta t)| & <\theta \mu a(t)\end{aligned}$ & $\begin{array}{l}b_{0}(t)=b_{0}(t-\Delta t) \\
\tilde{b}(t)=b(t)-b_{0}(t) \\
T(t)=M M D(\tilde{b}(t))\end{array}$ & ${ }_{-1}^{1 \uparrow} \prod^{D(\alpha)} \square \square$. \\
\hline
\end{tabular}

- Total sliding occurs when $a \geq 0$ and $|\tilde{b}| \geq \theta \mu a$. In this case the memory function equals \pm 1 on the whole interval $0 \leq \alpha \leq a$ and from Equation (3) it follows that the tangential load $|T|=\mu N$, in accordance to the Coulomb friction law. The total sliding distance is $b_{0}=b-\tilde{b}$ as one has to take into account the elastic deformation of asperities under tangential loading (i.e. $\tilde{b}= \pm \theta \mu a)$.

Table 1 contains a summary of the different criteria used to distinguish between the three contact states, with for each case an illustrative example of the memory function $D(\alpha)$.

\subsection{Friction-induced heat generation}

As a result of friction that occurs both during the partial slip and total sliding phases, energy will be dissipated in the form of heat. It has been shown that the dissipated energy can be found as the surface of the hysteretic loop that is generated during contact [10]. In case of a periodic normal and tangential loading, the hysteretic loop during each cycle will be the same and the amount of dissipated energy for each cycle will remain constant. Hence, the amount of total dissipated energy can be calculated as the product of the energy dissipated during one cycle and the number of cycles, as is done by Putignano et al. [11].

In the more general case of an arbitrary loading procedure, the dissipated energy can often not be calculated by considering hysteretic loop areas and other methods have to be developed. The MMD formulation turns out to be very useful for this purpose, as it was particularly developed to solve frictional contact problems for an arbitrary loading history. Indeed, the instantaneous friction-induced energy loss $\Delta W$ can be calculated as follows:

$$
\Delta W=-2 \mu(|\Delta b|-\theta \mu \Delta a) \cdot\left[N(a)-N(q)+\left.(q-a) \frac{d N}{d a}\right|_{a=q}\right]
$$


where all variables occurring are those that have been used in the mechanical contact model described above (i.e. normal and tangential displacements $a$ and $b$ and their increments $\Delta a$ and $\Delta b$, as well as normal load $N)$. A detailed analysis and derivation of this formula will be published elsewhere [12].

It is easily observed that the incremental energy loss $\Delta W$ is the product of a displacement factor and a load factor. The displacement factor contains the infinitesimal sliding distance $\Delta b$ taking into account the fact that contact of rough surfaces is not absolutely rigid in the tangential direction as asperities recede under tangential action, depending on the compression $a$. The load factor contains $N(a)$ and $N(q)$, which are the normal loads in case of a normal indentation of respectively $a$ and $q$. When describing contact between two flat profiles, there is no stick-slip boundary (i.e. $q=0$ ) and it is impossible to have non-zero $\Delta a$ during compression. Moreover, it can be shown that the derivative of the normal load to indentation is zero. Hence, the instantaneous energy loss equals $2 \mu|\Delta b| N(a)=2|\Delta b| T$, which is the known solution for work done by friction for two flat surfaces in contact.

\section{Numerical thermo-mechanical model for frictional contacts}

A successful model for the simulation of dynamically excited frictional contacts that is also capable of calculating heat generation at the contact interface requires three components: a crack model, a solid mechanics unit, and a thermal unit. The crack model should take into account the normal and tangential behavior at the contact interface, the solid mechanics unit is essential for solving the elastic wave equations and the thermal unit is required to solve the thermal diffusion equation. In this study, the elasticity equations are solved using the Structural Mechanics module [13] of the commercially available software package COMSOL Multiphysics ${ }^{\circledR}$, which is based on finite elements. The thermal diffusion problem is solved using the Heat Transfer module [14] from the same software package. COMSOL ${ }^{\circledR}$ also allows to use external user-supplied models [15]. This tool is used to implement the mechanical contact model described in the previous section at internal boundaries of the modeled geometry in the multiphysics software.

The link between the different components of the numerical model in a timedependent simulation is as follows:

(a) Calculation of displacements and temperature distribution

The Structural Mechanics module uses the normal and tangential stresses ( $\sigma$ and $\tau$ ), defined by appropriate internal and external boundary conditions, to calculate the displacements in the computational domain during each time step $t$ of the procedure. The normal and tangential displacements $\left(u_{n}\right.$ and $u_{t}$ ) on the contact interface are stored as output of the COMSOL ${ }^{\circledR}$ module, and used as input in the MATLAB ${ }^{\circledR}$ procedure. Using the multiphysics modeling capabilities of the COMSOL ${ }^{\circledR}$ software, a heat diffusion problem 
in response to an internal boundary heat source is solved at the same time using the Heat Transfer module in order to find the temperature distribution in the computational region.

(b) Calculation of contact stresses and energy losses

The displacement-driven contact model, implemented in MATLAB ${ }^{\circledR}$, is used to determine the new stress values at the integration points on the contact interface. At the same time, the friction-induced energy loss is calculated for each of these points. The calculated stress values and energy losses are then returned to $\mathrm{COMSOL}^{\circledR}$ in order to update the mechanical (i.e. internal thin elastic layer) and thermal (i.e. internal heat source) boundary conditions at the contact interface in respectively the Structural Mechanics and Heat Transfer modules. Note that in this step it is assumed that all energy "wasted" through friction is converted into heat.

(c) Repeated calculations

Steps (a) and (b) are repeated for the next time step $t+\Delta t$, until the desired calculation time is reached.

The above-mentioned approach is similar to the implementation described in more detail in Delrue et al. [9], however, here augmented with the thermal modeling part.

\section{Example: ultrasonic wave-crack interaction}

In order to illustrate the potential of the proposed multiscale thermo-mechanical model, an example of a propagating shear ultrasonic wave in a $2 \mathrm{D}$ aluminum sample with an internal crack is discussed. This example allows to reveal typical effects of contact interactions, such as nonlinear vibrations and friction-induced heating.

\subsection{Model specifications}

A rectangular aluminum sample with a height of $100 \mathrm{~mm}$ and a width of $50 \mathrm{~mm}$ is studied. The sample has a density $\rho=2700 \mathrm{~kg} \mathrm{~m}^{-3}$, a Young's modulus $E=$ $70 \mathrm{GPa}$ and a Poisson's ratio $\nu=0.33$. A crack of length $20 \mathrm{~mm}$ is introduced at the center of the sample, inclined over 20 degrees with respect to the horizontal. The friction coefficient $\mu$ has been set equal to one. On the top boundary, a $100 \mathrm{kHz}$ continuous shear wave excitation is applied. The other boundaries are defined as low reflecting boundaries in order to eliminate wave reflections at the edges of the computational region. At the internal crack, a thin elastic layer boundary condition is applied from the Structural Mechanics module and a boundary heat source is defined using the Heat Transfer in Solids module, as described in the previous section. The normal reaction curve that is required in the MMD crack model was taken from literature on ultrasonic assessment of properties of contact between two aluminum samples [16, 17]. 


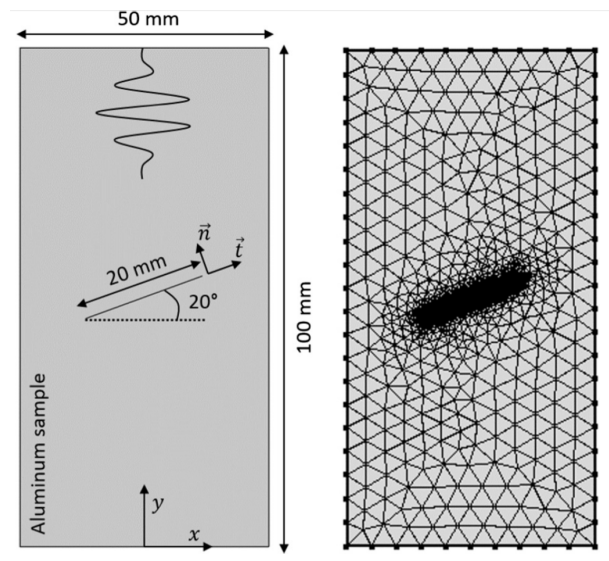

Fig. 2. Figure taken from [9] to illustrate the geometry of the model as implemented in $\mathrm{COMSOL}^{\circledR}$, together with the generated mesh. A rectangular aluminium domain contains a crack of $20 \mathrm{~mm}$, positioned in the center of the sample and inclined over 20 degrees (Taken from [9]).

Figure 2 shows the studied geometry, next to the full mesh of the geometry, using quadratic triangular elements. When studying elastic wave propagation problems, a minimum of six second order mesh elements per wavelength is needed. This corresponds to a maximum element size of $5.2 \mathrm{~mm}$. However, in the crack region, smaller elements are required to obtain an accurate and stable solution from the MMD algorithm. As described earlier, MMD approximates a crack by a number of mesoscopic cells. These should be small enough so that macroscopic elastic fields are uniform in each cell, but large enough to host a large number of asperities. In the finite element model, each mesh boundary on the crack interface represents a mesoscopic cell. In this particular example, the crack is divided into 150 mesh elements, each with a size of $0.13 \mathrm{~mm}$.

The implicit generalized alpha time-dependent solver is used to solve the wave propagation problem. As a rule of thumb, COMSOL ${ }^{\circledR}$ requires 20 time steps per cycle of the applied wave. However, MMD requires a higher temporal resolution to get accurate solutions. The MMD crack model causes local nonlinear vibrations at the crack interface, involving higher-order frequencies. In this example, 200 time steps per wave cycle are chosen, corresponding to a time step of $\Delta t=50 \mathrm{~ns}$.

\subsection{Normal and tangential stress-displacement relations}

Upon solving the model, the shear wave, excited with an amplitude of $100 \mathrm{~nm}$, will first straightforwardly propagate through the aluminum sample until it reaches the crack and starts interacting with it. Both dynamic clapping (i.e. repetitive opening and closing of the crack) and dynamic friction (i.e. rubbing of the crack faces against each other) will occur, which will result in nonlinear 

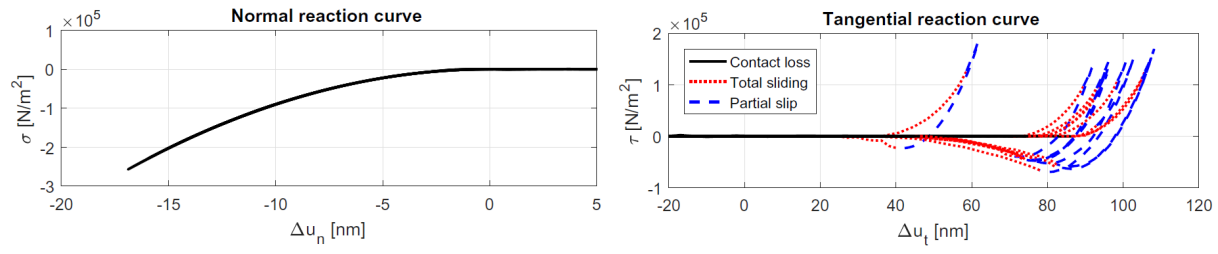

Fig. 3. The normal (left) and tangential (right) stress-displacement curves for the central point on the crack when excited by a shear wave excitation at $100 \mathrm{kHz}$ with an amplitude of $100 \mathrm{~nm}$. the normal reaction curve shows quadratic behavior in case of contact, while the tangential reaction curve shows hysteretic behavior with several loops while switching between the different contact states (Taken from [9]).

wave distortion as discussed in detail for the present example by Delrue et al. [9]. This behavior is evidenced by the nonlinear normal and tangential stressdisplacement relations calculated at the central point on the crack interface and depicted in Figure 3. The normal reaction curve clearly shows a quadratic relation between the normal contact stress and the relative normal displacement in case of contact. The tangential reaction curve, where switching between the three contact states can be identified, clearly indicates hysteresis behavior with several hysteresis loops due to the presence of friction occurring in the total sliding and partial slip regimes. Due to this hysteretic behavior, energy will be dissipated in the form of heat.

\subsection{Generation and diffusion of nonlinear features}

The nonlinear behavior associated with contact interaction and observed in the stress-displacement curves should results in the generation of nonlinear features at the crack interface, evidenced for instance by the appearance of harmonics of the excitation frequency. This is illustrated in Figure 4 where the frequency spectra of the relative normal and tangential displacements at 21 positions on the crack interface are shown. In order to accentuate the nonlinear components, the Scaling Subtraction Method (SSM) has been used [18]. SSM is based on the fact that only a high amplitude excitation can activate nonlinear defects in a sample, whereas a low amplitude excitation generates linear results. Hence, the low amplitude response can serve as a reference signal. The difference between the responses calculated after multiplication of the reference one by a proper amplification factor produces a nonlinear signature of damage. Here, a high amplitude excitation $A_{\text {high }}=100 \mathrm{~nm}$, and a low amplitude excitation $A_{\text {low }}=10 \mathrm{~nm}$ have been used. The generated harmonic frequencies in the resulting SSM signals for both the relative normal and tangential displacements are clearly visible. The harmonics that are present in the relative normal displacement signal are mainly caused by clapping effects (i.e. normal interaction between the crack faces), whereas the harmonic frequencies present in the relative tangential displacement signal are predominantly generated by friction effects (i.e. tangential 

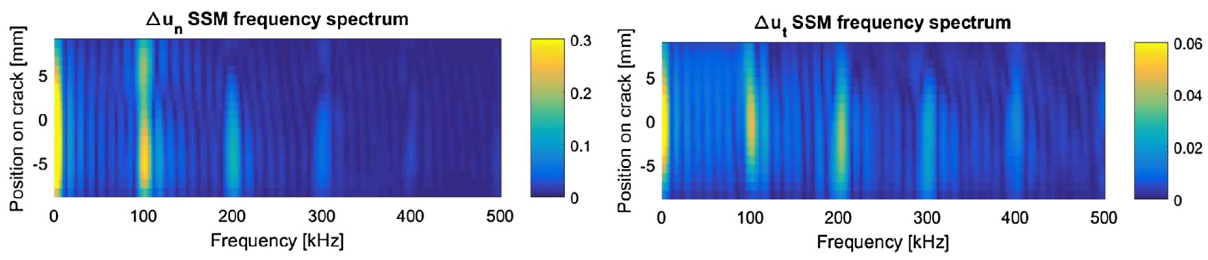

Fig. 4. Generation of higher order harmonics of the applied excitation frequency at the crack interface. The Fourier spectrum of the relative normal and tangential displacement signals $\left(\Delta u_{n}\right.$ and $\left.\Delta u_{t}\right)$ is calculated for 21 positions on the crack interface in case of a shear wave excitation at $100 \mathrm{kHz}$, after applying the Scaling Subtraction Method (SSM) [18]. The displacement signals were obtained by subtracting the displacements obtained at an amplitude $A_{l o w}=10 \mathrm{~nm}$ from the signals obtained at amplitude $A_{\text {high }}=10 A_{\text {low }}=100 \mathrm{~nm}$. Harmonic frequencies are mainly occurring at the left side of the crack interface (i.e. negative positions) (Taken from [9]).
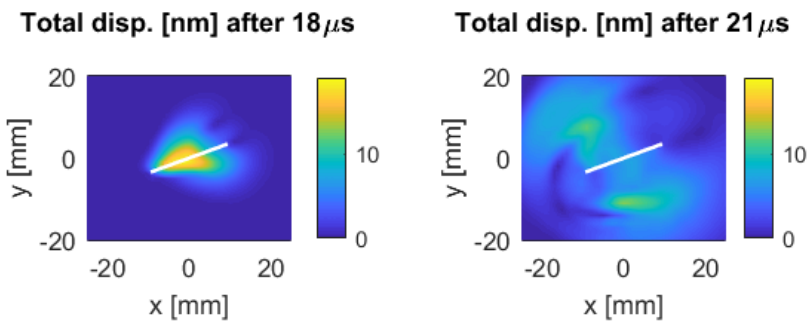

Fig. 5. Snapshots of the scale subtracted total displacement in the aluminum sample at two different instances in time illustrating the nonlinear wave propagation in the sample.

interaction between the crack faces). Note that due to the oblique incidence of the shear wave on the crack, harmonics are mainly observed at the left side of the crack interface (corresponding to negative positions).

In order to illustrate the dynamics of the contact model at the interface of the crack and the redistribution of the nonlinear signatures over the entire sample, Figure 5 illustrates the time evolution of the nonlinear wave propagation by means of two snapshots of the scale subtracted total displacement time signals in the aluminum sample. Once the first part of the shear wave has reached the crack $(t \approx 18 \mu \mathrm{s})$, the dynamic crack-wave interaction starts and nonlinear features are generated. Few microseconds later, the crack is in an open state and no contact interaction is observed. This means that the previously generated nonlinear features have propagated some distance in the sample, while generation of new nonlinear features is not detected. 


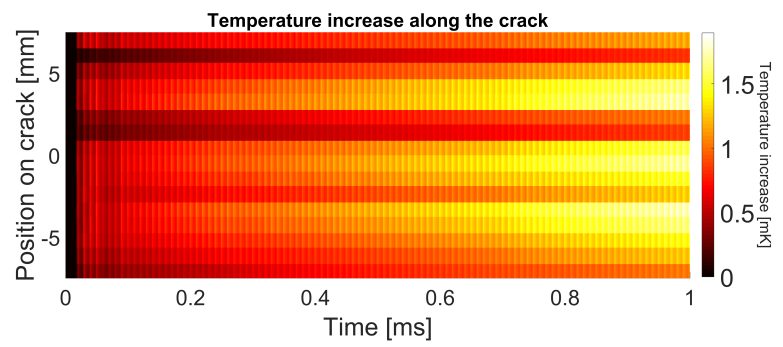

Fig. 6. Temperature increase at 21 points on the crack interface for a timespan of $1 \mathrm{~ms}$. Temperature increases during contact phases, while a local drop in temperature is observed when the contact is lost. In correspondence to Figure 4, heat generation is more pronounced at the left side of the crack, as friction effects are more efficiently generated there.

\subsection{Generation and diffusion of heat}

The hysteretic tangential reaction curve in Figure 3 revealed that heat should be generated at the crack interface due to friction effects in the partial slip and total sliding regimes. Figure 6 shows the temperature increase calculated at 21 positions on the crack interface, in correspondence to Figure 4. During each contact phase, heat is generated due to friction. When the contact is lost, heat diffuses away from the contact area and the temperature drops, as is observed from the alternations in the temperature at each contact point. After $1 \mathrm{~ms}$, the maximum temperature increase is $1.8 \mathrm{mK}$. As with the nonlinear generation, more heat is being generated on the left side of the crack. This was expected as nonlinear friction effects are also more pronounced on the left hand side of the crack.

Figure 7 illustrates the heat diffusion process by means of two snapshots of the temperature distribution in the aluminum sample. The first snapshot corresponds to the situation after $1 \mathrm{~ms}$, the last time instance considered in Figure 6, when the shear excitation is turned off. From then on, no new contact interactions occur and no additional heat is being generated. All heat present in the system now diffuses through the aluminum sample, as illustrated in the snapshot after $200 \mathrm{~ms}$.

\section{Conclusion}

A multiscale numerical model for structures with internal frictional contacts was presented. The development of such a model is crucial to increase the understanding of the typical behavior of dynamically excited frictional contacts in different structures. The proposed model uses the Method of Memory Diagrams (MMD), a mesoscopic frictional contact model for contact with rough surfaces. The full code allows to describe three different contact states (contact loss, total 

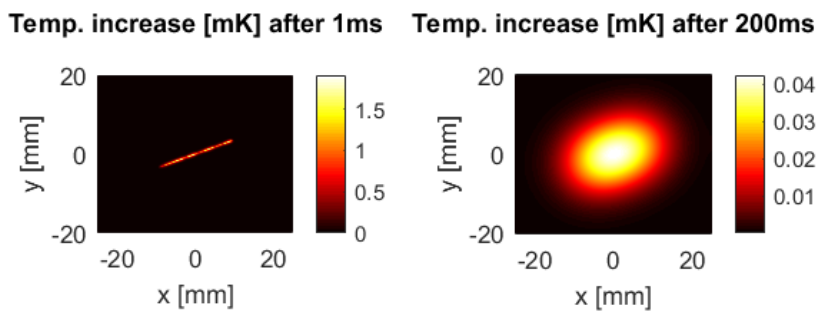

Fig. 7. Snapshots of the temperature distribution in the aluminum sample at two different instances in time, illustrating the heat generation at the crack interface and heat diffusion in the sample.

sliding and partial slip), as well as the problem of friction-induced heat generation, and is integrated into a commercially available finite element modeling environment (COMSOL $\left.{ }^{\circledR}\right)$. The supporting value of the final thermo-mechanical numerical model has been illustrated for nonlinear ultrasonic and thermosonic non-destructive testing of materials with internal contact defects. However, the developed model may also be of interest for researchers dealing with vibration of loaded contacts, nonlinear vibrometry, etc.

\section{Acknowledgements}

The research leading to these results has gratefully received funding from Internal Funds KU Leuven (C24/15/021).

\section{References}

[1] V.A. Yastrebov. Numerical methods in contact mechanics. Wiley-ISTE, 2013.

[2] P. Blanloeuil, A. Meziane, and C. Bacon. Numerical study of nonlinear interaction between a crack and elastic waves under an oblique incidence. Wave Motion, 51(3):425-437, 2014.

[3] R. Mindlin and H. Deresiewicz. Elastic spheres in contact under varying oblique forces. Journal of Applied Mechanics, 20:327-344, 1953.

[4] V.L. Popov and M. Heß. Method of Dimensionality Reduction in Contact Mechanics and Friction: a Users Handbook. I. Axially-Symmetric Contacts, volume 12. 2014.

[5] J Jäger. Axi-symmetric bodies of equal material in contact under torsion or shift. Archive of Applied Mechanics, 65(7):478-487, 1995.

[6] Michele Ciavarella. The generalized cattaneo partial slip plane contact problem. itheory. International Journal of solids and structures, 35(18):23492362, 1998. 
[7] V Aleshin, O Bou Matar, and Koen Van Den Abeele. Method of memory diagrams for mechanical frictional contacts subject to arbitrary $2 \mathrm{~d}$ loading. International Journal of Solids and Structures, 60:84-95, 2015.

[8] Vladislav Aleshin, Steven Delrue, Andrey Trifonov, Olivier Bou Matar, and Koen Van Den Abeele. Two dimensional modeling of elastic wave propagation in solids containing cracks with rough surfaces and friction - Part I: Theoretical background. Ultrasonics, 82:11-18, 2017.

[9] Steven Delrue, Vladislav Aleshin, Kevin Truyaert, Olivier Bou Matar, and Koen Van Den Abeele. Two dimensional modeling of elastic wave propagation in solids containing cracks with rough surfaces and friction Part II: Numerical implementation. Ultrasonics, 82:19-30, 2017.

[10] J.R. Barber, M. Davies, and D.A. Hills. Frictional elastic contact with periodic loading. International Journal of Solids and Structures, 48(13):20412047, jun 2011.

[11] C Putignano, M Ciavarella, and JR Barber. Frictional energy dissipation in contact of nominally flat rough surfaces under harmonically varying loads. Journal of the Mechanics and Physics of Solids, 59(12):2442-2454, 2011.

[12] Kevin Truyaert, Vladislav Aleshin, Steven Delrue, and Koen Van Den Abeele. Theoretical calculation of the instantaneous friction-induced energy losses in arbitrarily excited axisymmetric mechanical contact systems. Preprint, 2018.

[13] COMSOL, AB, Stockholm, and Sweden. Structural Mecanics Module User's Guide, COMSOL Multiphysics ${ }^{\circledR}$ v. 5.3 (2017).

[14] COMSOL, AB, Stockholm, and Sweden. Heat Transfer Module User's Guide, COMSOL Multiphysics ${ }^{\circledR}$ v. 5.3 (2017).

[15] COMSOL, AB, Stockholm, and Sweden. LiveLink ${ }^{T M}$ for MATLAB ${ }^{\circledR}$, COMSOL Multiphysics ${ }^{\circledR}$ v. 5.3 (2017).

[16] S Biwa, S Nakajima, and N Ohno. On the acoustic nonlinearity of solid-solid contact with pressure-dependent interface stiffness. TRANSACTIONSAMERICAN SOCIETY OF MECHANICAL ENGINEERS JOURNAL OF APPLIED MECHANICS, 71(4):508-515, 2004.

[17] Maodan Yuan, Jianhai Zhang, Sung-Jin Song, and Hak-Joon Kim. Numerical simulation of rayleigh wave interaction with surface closed cracks under external pressure. Wave Motion, 57:143-153, 2015.

[18] M Scalerandi, A S Gliozzi, C L E Bruno, and K Van Den Abeele. Nonlinear acoustic time reversal imaging using the scaling subtraction method. Journal of Physics D: Applied Physics, 41(21):215404, 2008. 